\title{
Antenna-Coupled Bolometer Arrays Using Transition-Edge Sensors
}

\author{
Michael J. Myers ${ }^{\mathrm{a}}$, Peter Ade ${ }^{\mathrm{b}}$, Greg Engargiola ${ }^{\mathrm{c}}$, William Holzapfel ${ }^{\mathrm{a}}$, Adrian T. Lee ${ }^{\mathrm{a}}$, Roger \\ O’Brient ${ }^{\mathrm{a}}$, Paul L. Richards ${ }^{\mathrm{a}}$, Andy Smith ${ }^{\mathrm{d}}$, Helmuth Spieler ${ }^{\mathrm{e}}$, Huan Tran ${ }^{\mathrm{a}, \mathrm{f}}$ \\ a) Department of Physics, University of California, Berkeley CA \\ b) Cardiff University, Physics Department, Wales \\ c) Department of Astronomy, University of California, Berkeley CA \\ d) Northrop Grumman Corporation, Redondo Beach CA \\ e) Physics Division, Lawrence Berkeley National Laboratory, Berkeley CA \\ f) Miller Institute, Berkeley CA
}

\begin{abstract}
We describe the development of an antenna-coupled bolometer array for use in a Cosmic Microwave Background polarization experiment. Prototype single pixels using double-slot dipole antennas and integrated microstrip band defining filters have been built and tested. Preliminary results of optical testing and simulations are presented. A bolometer array design based on this pixel will also be shown and future plans for application of the technology will be discussed.
\end{abstract}

Keywords: Antenna-coupled, bolometer, array, TES

\section{INTRODUCTION}

Bolometers are the most sensitive photometric detectors of millimeter wavelength light. They have found significant astronomical applications in this area, such as the characterization of the Cosmic Microwave Background anisotropy and galaxy cluster surveys. Improvements in detector technology have historically increased the observational power of these experiments by lowering detector noise, but current Transition-Edge Sensor (TES) based bolometers are at the photon noise limit for many of these applications. Therefore, little is to be gained by reducing the detector noise further, and for future experiments requiring more sensitivity increasing the size of the detector array is the only clear avenue to pursue. We are developing these detectors to study the polarization of the Cosmic Microwave Background.

Antenna-coupled bolometers ${ }^{1}$ provide a new level of integration that is critical for the construction of large bolometer arrays. A receiver using traditional absorber-coupled bolometers will often use large scalar horns to define the beam seen by the detector. Quasioptical band defining filters are also necessary and system complexity can grow significantly if multiple bands are desired. With an antenna-coupled bolometer, these elements can be integrated into the detector chip itself. A planar antenna can be chosen which has a convenient pattern to match to the telescope optics, removing the need for bulky horns. The band defining filters can be integrated on-chip as part of the transmission line used to feed the antenna. Antennas can also be polarization sensitive, which is advantageous in our application. Use of a broadband antenna would allow a single pixel to detect several different frequency bands.

\section{PROTOTYPE PIXEL}

We have designed and fabricated a test chip including prototype pixels for the bolometer array. The preliminary optical test results for these chips are presented.

\subsection{Design}

Our single pixel design uses a double-slot dipole antenna resonant at $217 \mathrm{GHz}$ combined with a silicon hyperhemispherical lens. This well studied design ${ }^{2}$ has several attractive features, such as its symmetric beam pattern and linear polarization. A dual linear polarization version of this antenna also exists ${ }^{3}$. This silicon lens narrows the antenna beam to make it easier to couple to a typical telescope, as well as greatly reducing the problem of substrate mode excitations. 


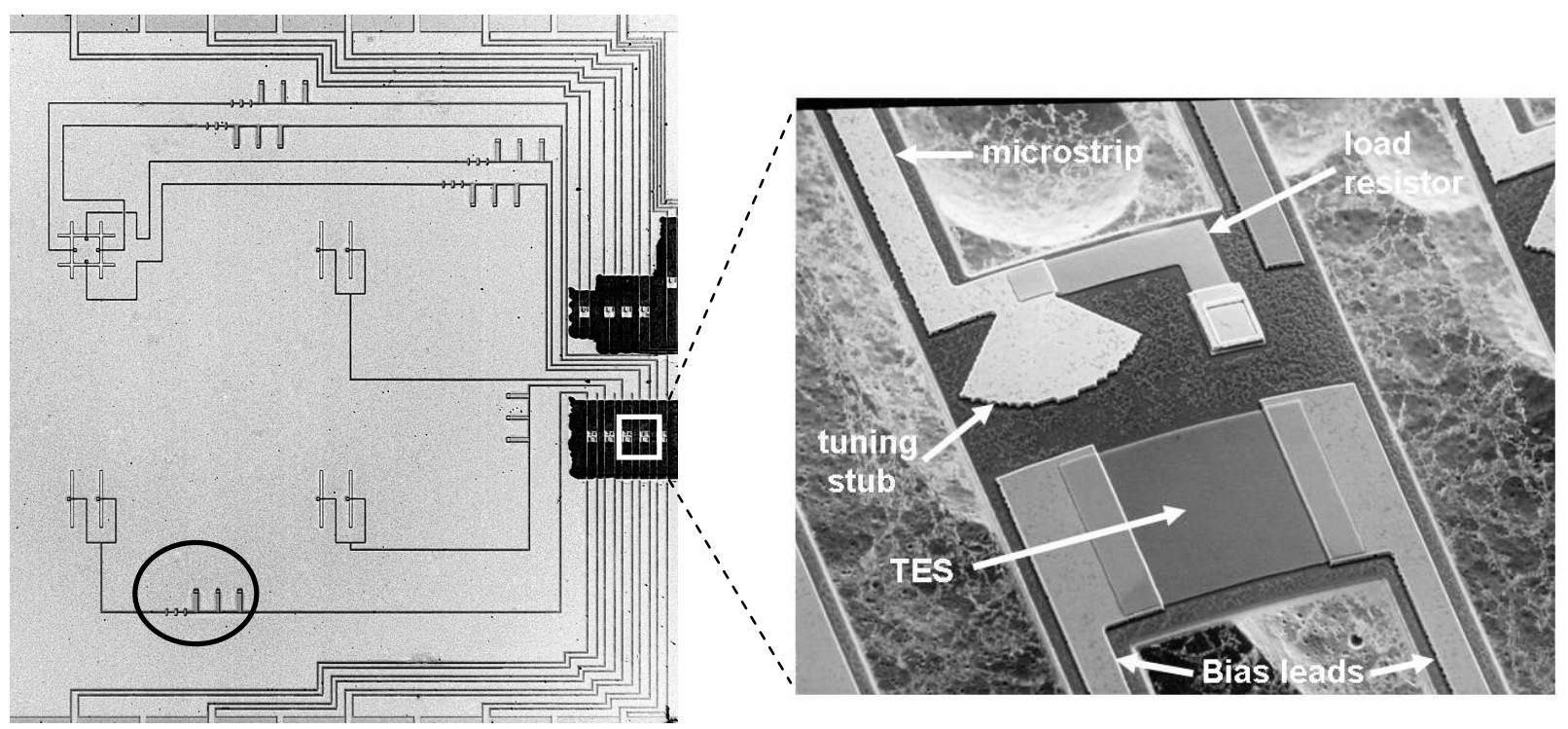

Figure 1: Left - Photo of antenna coupled bolometer test chip. The four different slot antennas are visible on the left half of the photo. The microstrip bandpass and lowpass filters one on of the devices are circled. The upper left slot antenna is the dual polarization variant. On the far right the nitride bridges suspending the TESs and load resistors are visible. The dark area around them is the silicon that has been etched to suspend the bolometers.

Right - Close-up of nitride bridge. This detector has a radial stub to tune out the inductance of the load resistor. The stub was not present on the devices currently being reported.

The antenna is fed by a superconducting microstrip transmission line. Superconducting transmission lines generally have low conductor loss up to a frequency corresponding to twice the gap energy in the material ${ }^{4}$. This frequency is about $700 \mathrm{GHz}$ in niobium, making it well suited as a microstrip conductor for our purpose. The dielectric loss will depend upon the quality of the material deposited.

At the antenna, the microstrip is sized to match the antenna impedance of $30 \Omega$. This results in a fairly narrow line width of $2.7 \mu \mathrm{m}$ given our conductor and dielectric thicknesses. It is convenient to have a larger line width at the filters in order to minimize the effect of possible variations in line width on the filter performance. A tapered impedance transformer ${ }^{5}$ is used to bring the line impedance down to $10 \Omega$, which corresponds to a $9.8 \mu \mathrm{m}$ wide microstrip.

Once the line impedance is $10 \Omega$, it enters the band defining microstrip filters. The bandpass filter used here is the well known resonant design using $1 / 4$ wavelength shorted stubs ${ }^{6}$. This filter was chosen because the line impedances required correspond to microstrip dimensions which are straightforward to fabricate. It is also conceptually simple. This type of filter has a well defined passband, but also has undesirable satellite bands at odd integer multiples of the design band due to the periodicity of the structure. A stepped-impedance low pass filter ${ }^{7}$ is used to suppress the higher frequency bands while leaving the lowest passband unaffected.

Initial filter design was done using $\mathrm{MMICAD}^{8}$, a linear transmission line circuit simulator. The circuit geometry was then generated using a published model for superconducting microstrip ${ }^{9}$. This geometry was then modeled in Sonnet ${ }^{10}$, a full wave electromagnetic simulator, using a surface inductance correction for the niobium ${ }^{11}$. A strong agreement is seen between the simplest MMICAD models and the full wave analysis, indicating that the transmission line circuits should act much like the ideal case.

After the band defining filters, the microstrip is terminated into a load resistor which is thermally isolated on a silicon nitride bridge. A TES adjacent to the load resistor on the nitride bridge measures the dissipated power. 
The entire microstrip circuit is simulated by modeling everything except the antenna in Sonnet, as the boundary conditions assumed in Sonnet are not well suited for antenna simulation. The impedance matrix for the circuit was then exported and combined with the antenna impedance, which was taken from published values ${ }^{12}$ and verified with IE $3 \mathrm{D}^{13}$. This yields a total circuit model which can be compared with the measured response of the detector.

Each test chip includes four pixels: one with no microstrip filters, one with just the resonant bandpass filter, one with both the bandpass filter and the stepped impedance low pass filter, and the last with the dual polarization version of the double slot antenna along with both band defining filters.

\subsection{Fabrication}

Our process begins with the deposition of $1 \mu \mathrm{m}$ of low stress silicon nitride onto a standard 4-inch silicon wafer using low pressure chemical vapor deposition. A $0.3 \mu \mathrm{m}$ niobium ground plane is sputter deposited. The ground plane is then patterned using a 10x reduction wafer stepper and $1 \mu \mathrm{m}$ of i-line photoresist. The ground plane is etched using a Reactive Ion Etch (RIE) system. This layer acts as both the conductor for the slot antenna and as the ground plane for the superconducting microstrip. A $0.5 \mu \mathrm{m}$ silicon dioxide dielectric layer is deposited using a plasma enhanced chemical vapor deposition system. This layer is patterned as before and again etched in the RIE system using a different etch recipe suited for $\mathrm{SiO}_{2}$. A $0.6 \mu \mathrm{m}$ thick niobium layer for the microstrip is deposited, patterned, and etched.

A bilayer consisting of $400 \AA$ aluminum and $800 \AA$ titanium is then deposited in one vacuum step to form the TES. An argon sputter etch is necessary before the deposition to ensure superconducting contact between the niobium leads and the TES. The $\mathrm{T}_{\mathrm{C}}$ of the Al/Ti bilayer deposited in this machine has proven very repeatable and easily tunable ${ }^{14}$ in the range of interest for use with a $300 \mathrm{mK}$ refrigerator $(400-600 \mathrm{mK})$. This layer is also used to make the load resistor terminating the microstrip line. Since the energy of photons at $200 \mathrm{GHz}$ is much larger than the gap energy of the $\mathrm{Al} / \mathrm{Ti}$ bilayer, this material can be used as the load resistor as well, saving an additional metallization layer. The layer is patterned and etched using an $\mathrm{SF}_{6}$ plasma etch followed by a standard wet $\mathrm{Al}$ etch, which does not damage the $\mathrm{Nb}$ and $\mathrm{SiO}_{2}$ beneath.

With the electrically active components finished, the low stress silicon nitride is patterned to allow release of the nitride bridge. The wafer is diced using a wafer saw and the chips are etched in a gaseous $\mathrm{XeF}_{2}$ etcher. This etch safely removes the silicon from underneath the nitride bridge providing the required thermal isolation for the TES and load resistor.

After a final $\mathrm{O}_{2}$ plasma etch to remove the photoresist, the chip has a $0.1 \mu \mathrm{m}$ gold layer evaporated on part of the back of the wafer directly under the nitride bridge using a shadow mask. This is done to prevent direct stimulation of the TES while not interfering with the antenna beam.

\subsection{Test Apparatus}

We have constructed a cryogenic system for optical testing of these detectors. The detector is mounted to the silicon lens and attached to a copper plate. This plate is thermally isolated from the ${ }^{4} \mathrm{He}$ cold plate by three vespel rods. A copper heat strap then connects the detector mounting plate to the cold head of a single stage closed cycle ${ }^{3} \mathrm{He}$ cooler. In this configuration, the detector stage reaches a base temperature of $295 \mathrm{mK}$. An aluminum shield covers the back side of the detector to protect it from direct stimulation by stray light.

The silicon lens faces a TPX plastic lens which is used to reimage the detector focus at the dewar shell, minimizing the required size of the optical window and filters. An $18 \mathrm{~cm}^{-1}$ metal mesh low pass filter at $77 \mathrm{~K}$ and an alkali halide filter at $4 \mathrm{~K}$ are used for IR blocking. A $1.3 \%$ neutral density filter is also mounted at $4 \mathrm{~K}$ to prevent $300 \mathrm{~K}$ photons from saturating the detector, which has been designed to accommodate the atmospheric loading of a ground based CMB experiment. A Zotefoam optical window is used to bring light into the cryostat without breaking vacuum.

The optics used in the test receiver are roughly similar to what would be used in an ground based observational environment. The performance of the detectors in this system can be used to gauge what should be achievable in an actual observing receiver. 


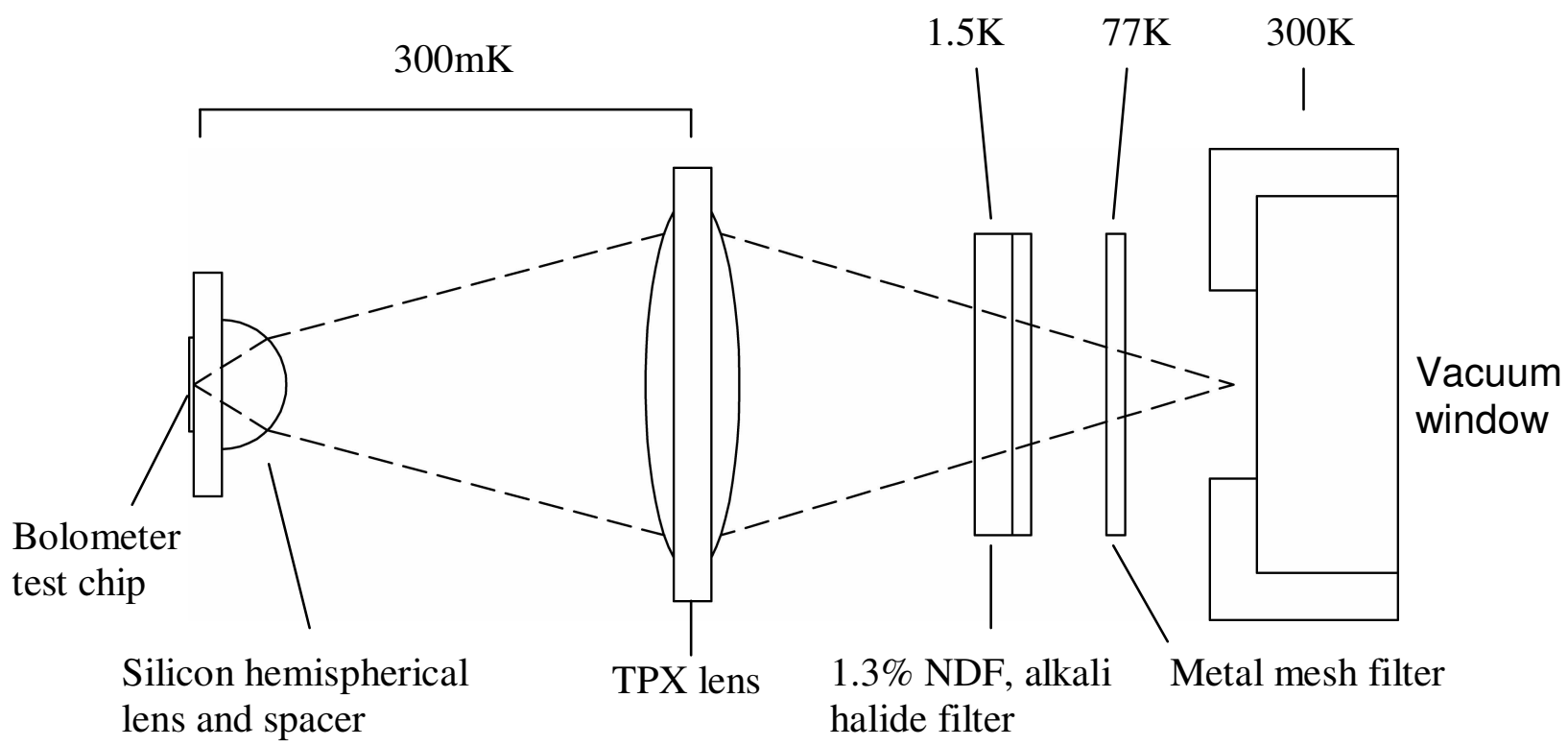

Figure 2: Schematic diagram of optics used in our bolometer test cryostat and the temperatures of each element (not to scale)

\subsection{Test Results}

We have electrically tested several devices and found them to function properly. Typical device parameters are a $\mathrm{T}_{\mathrm{C}}$ of $450 \mathrm{mK}$ and a thermal conductance $\mathrm{G}=9 \times 10^{-10} \mathrm{~W} / \mathrm{K}$. The electrical time constants are $\sim 0.2 \mathrm{~ms}$. The load resistor terminating the microstrip can not be nondestructively directly measured, but test structures on the chip indicate that the load resistor is $25 \Omega$. This deviation from the design value of $10 \Omega$ was caused by significant undercutting during the wet $\mathrm{Al}$ etch. This can be corrected in future fabrication runs by changing the etch conditions and modifying the resistor geometry to make it less sensitive to overetch. The microstrip width at the antenna was also found to be wider than desired, which can be corrected with a mask revision.

The detectors were voltage biased using a standard TES bias circuit ${ }^{15}$ with a $0.02 \Omega$ bias resistor. A Quantum Design SQUID was used to read out the current. The large input coil inductance of the Quantum Design SQUID was troublesome for this set of detectors due to the L/R time constant of the bias circuit being comparable to the electrical time constant of the device. Ideally the bolometer time constant should be somewhat longer than that of the bias circuit in order to maintain stability. A superconducting transformer was used to reduce the effective inductance in the bias circuit. This stabilized the system, but the resulting reduction in signal made the detector noise difficult to measure. An electrical noise plot for a previous device which did not require the transformer is shown in Figure 3. The earlier devices were near the white thermal fluctuation noise limit over the frequency range of interest for our experiment. Because the current devices were manufactured using the same process as the previously tested detectors, we expect similar noise performance. The stability of the earlier device without the transformer is due to its lower thermal conductance, giving it a longer time constant. SQUIDs with lower input coil inductances are available and would resolve this issue.

We also present some preliminary results of optical testing of two of these detectors. A rough beam map was made for one of the detectors using a Gunn oscillator source. The beam is symmetric as expected and the FWHM beam width of $4^{\circ}$ agrees with published data to within the tolerances of the measurement ${ }^{2}$. The polarization sensitivity was tested using a chopped liquid nitrogen load and a wire grid polarizer. The signal varied as expected with the angular position of the polarizer, with a cross-polarization measured at $3 \%$ of the co-polarization. This performance is likely limited by the test apparatus, which has not been optimized for good polarization performance. 


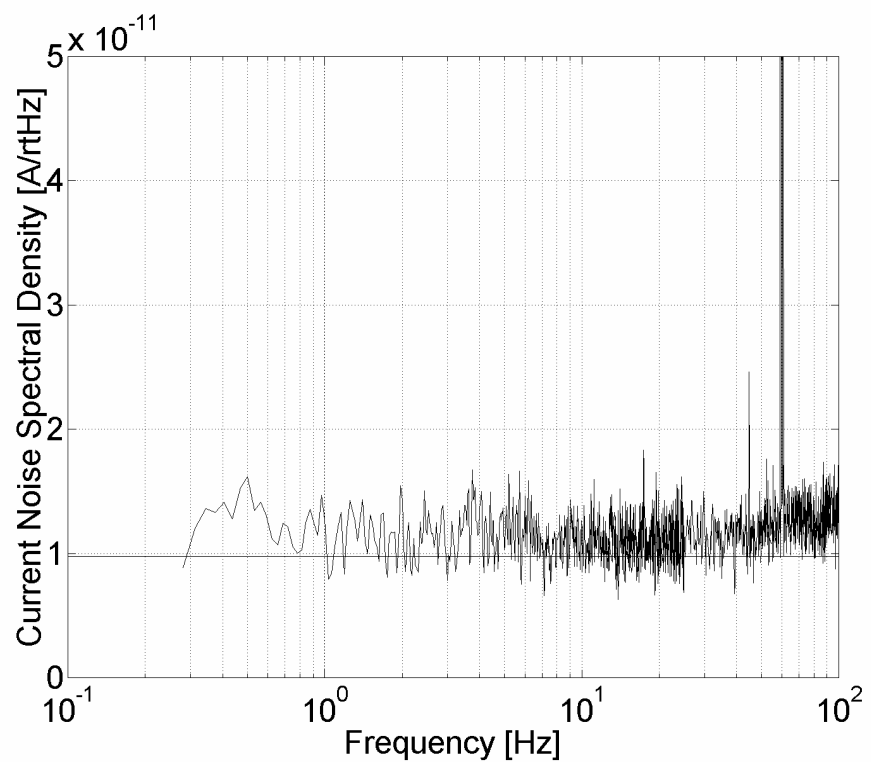

Figure 3: Measured noise for an antenna-coupled bolometer compared to the expected phonon noise (line). The measured noise is close to the expected phonon noise (line) from $100 \mathrm{~Hz}$ to well below $1 \mathrm{~Hz}$. The slight deviation is likely due to the inexact calculation of the detector bias voltage and thermal conductance used. A $60 \mathrm{~Hz}$ peak is visible due to power line coupling into the system. Above $100 \mathrm{~Hz}$ the noise increases, due at least in part to a peak in the SQUID readout system which is not detector related.

The spectral response of the detectors was measured using a Fourier Transform Spectrometer (FTS). The results of this measurement are shown in Figure 4 for a device with no microstrip filters. The response of a simple model including only on-chip frequency dependent effects is also shown. The spacing of the narrow peaks in the measured spectrum closely matches that in the model, so they can be assumed to be the result of standing waves on the transmission line. A reasonable agreement is seen in the peak to trough ratio across the spectrum. This indicates that the measurement is consistent with the expected microstrip termination mismatch due to the correctable fabrication issues. Other off-chip optical effects are not yet included in the simulation and may further improve the fit.

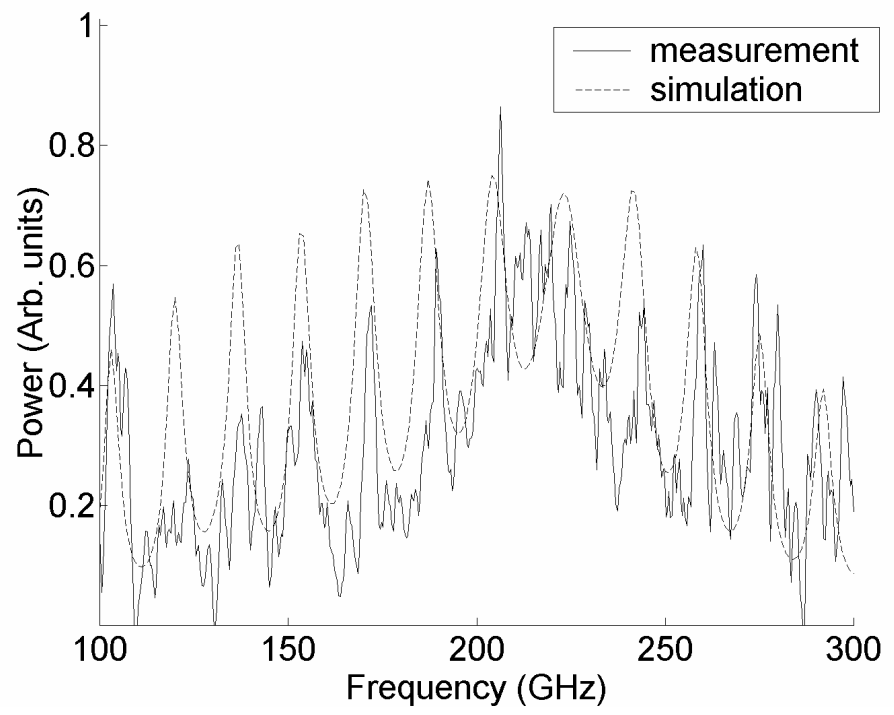

Figure 4: Measured spectral response in arbitrary units for an antenna coupled bolometer with no microstrip filters. The dotted line is the circuit simulation given the known impedance mismatches. 
A device with an on-chip microstrip bandpass filter was also optically tested with a FTS and the results are shown in Figure 5. The normalization of the spectrum to total receiver efficiency was done with a thermal load, chopped between $300 \mathrm{~K}$ and $77 \mathrm{~K}$ Eccosorb ${ }^{16}$.

The filter band is approximately $30 \%$ narrower than expected. We are currently working on fitting our model to the data for this device. Further testing will likely make the data analysis more straightforward.

We have made a preliminary comparison of the peak efficiency of the receiver to the expected value. The significant sources of optical loss in the test system include reflection at the surface of the silicon and TPX lenses and loss in the quasioptical low pass filters. On-chip loss is due to the impedance mismatch and loss in the microstrip. We estimate the total optical loss at $57 \%$ and the on-chip loss at $40 \%$, yielding an expected peak receiver efficiency of $26 \%$. The results seen in Figure 5 indicate a peak efficiency within 25\% of the expected value.

A possible source of the extra loss is vignetting of the optical beam in the test system, which will be investigated with further testing. Another potential source of loss would be higher than expected loss in the microstrip transmission lines. This will be investigated as well, however in our array design the microstrip lines will be significantly shorter than in this test chip, which would make the current level of excess loss negligible.

A known source of loss in the optics is the loss of $30 \%$ of the incoming power due to reflection at the uncoated silicon lens. A $1 / 4$ wavelength antireflection coating should reduce this drastically. Simulations of the microstrip circuit indicate a loss of at least another $30 \%$ due to the impedance mismatch on the chip. With these two sources of loss understood and correctable, we believe we can improve the total receiver efficiency by a factor of two. This would put this system's efficiency well within the typical range for receivers that have been used in actual observations.

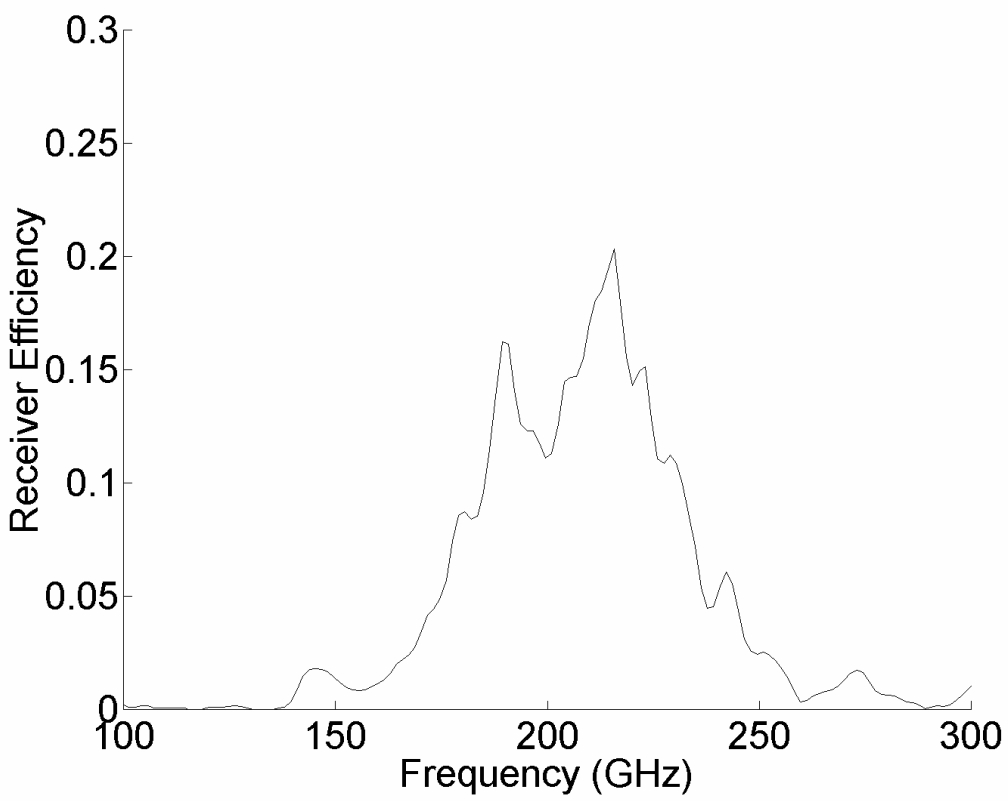

Figure 5: Measured end-to-end receiver efficiency using an antenna coupled bolometer with a bandpass filter. The data shown here was taken on a Fourier Transform Spectrometer with lower resolution than the one used for Figure 4. 


\section{ANTENNA-COUPLED BOLOMETER ARRAYS}

We have demonstrated a functioning single antenna-coupled bolometer pixel. We discuss the construction of an array of antenna-coupled bolometers and consider further possibilities for advances in array design.

\subsection{Current Bolometer Array Design and Fabrication}

We have designed an array of antenna-coupled bolometers based on the prototype pixel design. Since the pixel requires only iterative changes to be usable, we can begin construction of an array based on this design shortly.

Our array design is pictured in Figure 6. The first array will fit on a standard 4-inch wafer for convenience. This design has one frequency band per antenna so pixels designed for different frequencies are included to yield multicolor sensitivity. Since the antenna and resonant bandpass filters simply scale with frequency, we are confident that the same design can be used over different frequency ranges by simply modifying the appropriate dimensions. The highest frequency pixels will be the most sensitive to optical aberrations in the telescope, which will be worst at the edge of the focal plane. To make the best use of the focal plane, the array is designed with the highest frequency pixels at the center of the array and the lowest frequency pixels at the edge.

\subsection{Future Bolometer Array Designs}

Larger bolometer arrays based on our current pixel design can be constructed by arranging 4-inch wafer segments in pie-like wedges or by using a 6-inch wafer process, which is currently becoming available at the UC Berkeley Microlab. An array combining both techniques could accommodate over 1000 pixels.

Our current design uses a double-slot dipole antenna. This was chosen because it is a proven design which is well suited for our purposes. One disadvantage is the limited bandwidth of this resonant antenna. A broadband antenna would allow the construction of multicolor pixels, yielding the most efficient use of the focal plane possible. A wide variety of microstrip antenna designs exist, but achieving broadband performance while retaining the advantages of the current design is a challenge.

Another interesting possibility is the use of a phased antenna array to synthesize an antenna beam convenient for coupling to a telescope. A promising design of this type has been developed by the Caltech group ${ }^{17}$. This would eliminate the lens array necessary for our current array design, easing fabrication and making antireflection coating easier. One advantage with the use of a lens array, however, is the substantial space left around each antenna. This allows the placement of the entire microstrip circuit and TES immediately next to each pixel (see Figure 6), bringing only low frequency readout lines to the perimeter of the array. This minimizes the effect of loss in the microstrip and ensures that the wiring will not interfere with the efficient use of the focal plane.

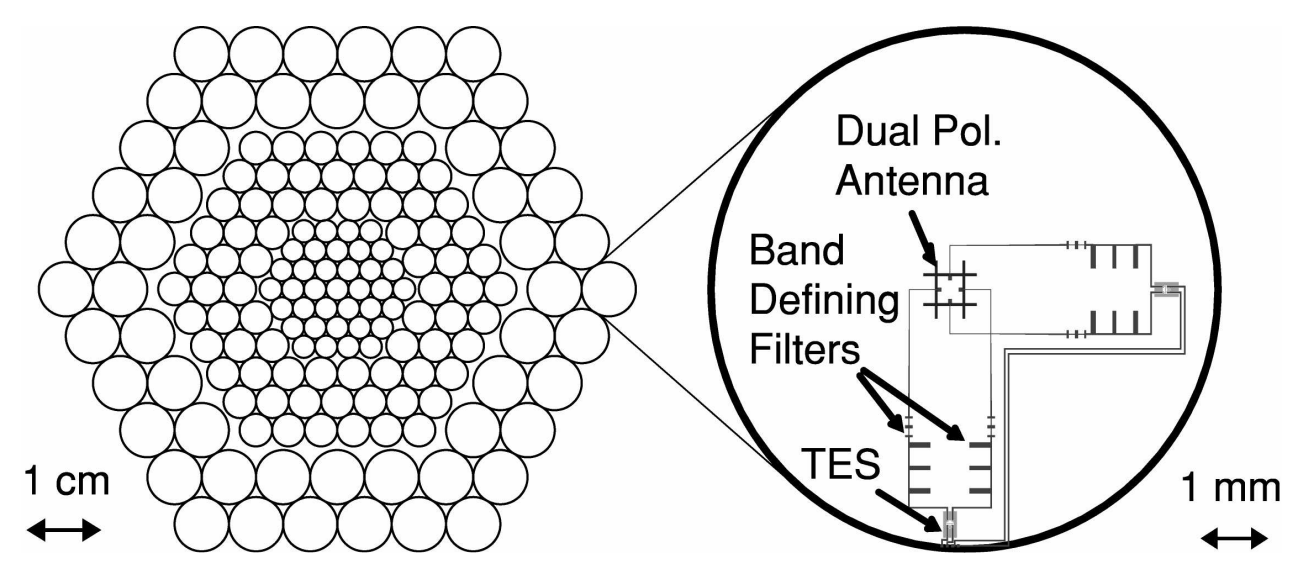

Figure 6: An antenna-coupled bolometer array based on our prototype pixel design. On the left is the full silicon lens array with 3 bands: $90 \mathrm{GHz}, 150 \mathrm{GHz}$, and $220 \mathrm{GHz}$. At right is the mask drawing for a single $90 \mathrm{GHz}$ pixel. 


\section{CONCLUSIONS}

We have designed, fabricated, and tested an antenna-coupled bolometer suited for large array construction. The detector functions largely as expected and, with the correction of a fabrication issue, we believe these devices can be used in an observational array. Further investigation into models of bolometer performance will be carried out.

We present a bolometer array based on our test pixel design and array construction will begin soon.

\section{ACKNOWLEDGEMENTS}

The authors would like to that Xiaofan Meng for his significant assistance in the fabrication of the devices described here. Many other UC Berkeley Microlab staff members were also supportive. This work was funded in part by NSF grant AST-0096933.

\section{REFERENCES}

1. Nahum M., Richards PL., "Design Analysis of a Novel Low Temperature Bolometer," IEEE Trans. on Magnetics, 27, pp.2484-7, March 1991

2. Filipovic D., Gearhart S., Rebeiz G., "Double-Slot Antennas on Extended Hemispherical and Elliptical Silicon Dielectric Lenses," IEEE Trans on Microwave Theory and Tech., 41, pp.1738-49, 1993

3. Chattopadhyay G., Zmuidzinas J., "A Dual-Polarized Slot Antenna for Millimeter Waves", IEEE Trans. On Antennas and Propogation, 46, pp.736-7, 1998

4. Kautz R.L., "Picosecond Pulses on Superconductive Striplines", J. of Appl. Phys., 49, pp.308-14, 1978

5. McGinnis D., Beyer J., "A Broad-Band Microwave Superconducting Thin-Film Transformer", IEEE Trans. On Microwave Theory and Tech., 36, pp.1521-5, 1988

6. Matthaei G., Young L., Jones E.M.T., Microwave Filters, Impedance-Matching Networks, and Coupling Structures, Sec. 10.03, Artech House, Dedham, Mass., 1964

7. Pozar D., Microwave Engineering, Sec. 8.6, Wiley, New York, 1998

8. MMICAD, Optotek LTD, http://www.optotek.com

9. Yassin G., Withington S., "Electromagnetic Models for Superconducting Millimeter-Wave and SubmillimeterWave Microstrip Transmission Line”, J. of Phys. D: Appl. Phys., 28, pp.1983-1991, 1995

10. Sonnet em Suite, Sonnet Software, Inc., http://www.sonnetusa.com

11. Kerr A.R., "Surface Impedance of Superconductors and Normal Conductors in EM Simulators", ALMA Memo No. 245, http://www.alma.nrao.edu/memos/

12. Zmuidzinas J., LeDuc H.G., "Quasi-Optical Slot Antenna SIS Mixers", IEEE Trans on Microwave Theory and Tech., 40, pp.1797-1804, 1992

13. IE3D, Zeland Software, http://www.zeland.com/

14. Werthamer, N.R., "Theory of the Superconducting Transition Temperature and Energy Gap Function of Superposed Metal Films", Phys. Rev., 132, pp.2440-2445, 1963

15. Lee A.T., Richards P.L., Nam S.W., Cabrera B., Irwin K.D., "A Superconducting Bolometer with Strong Electrothermal Feedback", Appl. Phys. Lett., 69, pp.1801-3, 1996

16. Emerson and Cuming Microwave Products, http://www.eccosorb.com/

17. Goldin A., Bock J.J., Hunt C.L., Lange A.E., LeDuc H.G., Vayonakis A., Zmuidzinas J., "Design of Broadband Filters and Antennas for SAMBA”, Proc. SPIE, 4855, pp.163-71, 2003 


\section{DISCLAIMER}

This document was prepared as an account of work sponsored by the United States Government. While this document is believed to contain correct information, neither the United States Government nor any agency thereof, nor The Regents of the University of California, nor any of their employees, makes any warranty, express or implied, or assumes any legal responsibility for the accuracy, completeness, or usefulness of any information, apparatus, product, or process disclosed, or represents that its use would not infringe privately owned rights. Reference herein to any specific commercial product, process, or service by its trade name, trademark, manufacturer, or otherwise, does not necessarily constitute or imply its endorsement, recommendation, or favoring by the United States Government or any agency thereof, or The Regents of the University of California. The views and opinions of authors expressed herein do not necessarily state or reflect those of the United States Government or any agency thereof or The Regents of the University of California. 